\title{
DIVERSIDAD Y CONSERVACIÓN DE LOS MAMÍFEROS DE UCAYALI, PERÚ
}

\section{DIVERSITY AND CONSERVATION OF MAMMALS OF UCAYALI, PERU}

\author{
Heidi Quintana ${ }^{1}$, Víctor Pacheco ${ }^{1,2}$ y Edith Salas ${ }^{1}$
}

\begin{abstract}
Resumen
El Perú reporta una alta diversidad de mamíferos, sin embargo no existe al presente un departamento o región con una lista actualizada de mamíferos. Por ello, se presenta aquí una lista actualizada para Ucayali, la que incluye 192 especies, en 11 órdenes y 35 familias (38\% de la mastofauna peruana). Del total de especies, 56 son registradas en Áreas Protegidas y 189 especies en Áreas No Protegidas. En Ucayali se encuentran tres especies endémicas para el país: la ardilla Sciurus pyrrhinus, el ratón cricétido Neusticomys peruviensis y el ratón equímido Makalata rhipidura. Se encontraron 86 localidades diferentes con registros de especímenes (la mayoría ubicados a lo largo de ríos). Se identificaron dos zonas con pocos o ningún registro denominadas “vacíos de información”: 1) la zona Nor Oriental de Ucayali (parte de la Reserva Nacional Sierra del Divisor), y 2) la zona oriental de Ucayali, conformada por el territorio cruzado por el río Yucanya, el río Yurúa y, la cuenca baja del río Purús (parte del Parque Nacional Alto Purús). Estas zonas son recomendadas para estudios de biodiversidad y conservación.

Palabras clave: Mamíferos, diversidad, Ucayali, base de datos, conservación, vacíos de
\end{abstract} información

\begin{abstract}
Peru reports a very high diversity of mammals; however no Peruvian department or region has an updated list of mammals to date. Therefore, we present an updated list for Ucayali, which includes 192 species, belonging to 11 orders and 35 families (comprising 38\% of all Peruvian mammals). Of these, 56 are recorded in protected areas and 189 species in unprotected areas, respectively. Three endemic species for the country were found in Ucayali: Sciurus pyrrhinus, a squirrel, Neusticomys peruviensis a cricetid mouse, and Makalata rhipidura an echimyd rodent. 86 different locations had specimen records (mostly located along rivers). Two areas with scarce or lack of records were found and rated as "Information gaps": 1) northeast of Ucayali (part of the Sierra del Divisor National Reserve), and 2) eastern Ucayali, composed of the territory crossed by the Yucanya River, the Yurua River, and downstream Purus River (part of the Alto Purus National Park). These areas are recommended for biodiversity and conservation studies.
\end{abstract}

Key words: Mammals, diversity, Ucayali, database, conservation, information gaps

\section{Introducción.}

El Perú es uno de los cinco países a nivel mundial con mayor diversidad de mamíferos, estimándose que no menos de 508 especies de mamíferos se distribuyen a lo largo del territorio peruano (Pacheco et al., 2009). Esta diversidad varía con la geografía del territorio peruano incrementándose en la selva baja tropical (Voss \& Emmons, 1996; Emmons \& Feer, 1997; Pacheco, 2002); no obstante, se conoce poco sobre el estado actual de la diversidad de mamíferos a nivel regional, lo cual limita la toma de decisiones en cuanto a las prioridades de conservación.

Ucayali perteneció inicialmente a la jurisdicción del departamento Loreto, formalizándose su autonomía el 18 de junio de 1980 por Decreto Ley N ${ }^{\circ}$ 23099 sobre las bases de las provincias de Ucayali y Coronel Portillo, y por Ley $\mathrm{N}^{\mathrm{o}} 23416$ del 01 de junio de 1982 quedó conformado por cuatro provincias: Coronel Portillo, Atalaya, Padre Abad y Purús. Así también, el 25 noviembre de 1988, se creó la región
Ucayali sobre las bases de la Ley Orgánica de Creación No 24945.

Las primeras investigaciones de la diversidad de mamíferos en la región fueron realizadas a mediados del siglo XIX, por Deville y Castelnau (Gervais, 1855) y Edward Bartlett en 1860 (Bartlett, 1871). A principios del siglo $\mathrm{XX}$, comienzan las primeras evaluaciones significativas con Latham Rutter quien realizó una colecta de 157 especímenes (Thomas, 1924; 1928) y Russell W. Hendee con un inventario de más de 300 mamíferos (Thomas, 1928). La primera compilación para la región, basada en 341 especímenes, de las cuales 18 fueron primeros registros, se remonta a Sanborn (1949); apareciendo luego algunos estudios más específicos, como la colecta de quirópteros en el río Ucayali (Dorst, 1953) y la revisión de primates del género Lagothrix (Fooden, 1963). A partir de 1963, J. O’Neill empieza a registrar especímenes de mamíferos en la localidad de Balta, registros que se complementan con colectas 
Julio - Diciembre 2009

intensivas realizadas por Alfred L. Gardner (junioagosto 1966, junio-agosto 1968, marzo-abril 1971) y James L. Patton (junio-agosto 1968); culminando con el registro de 130 especies de mamíferos para la localidad de Balta (Voss \& Emmons, 1996), una de las localidades más diversas a nivel mundial.

A finales del siglo XX, se publican observaciones sobre la flora y fauna de los ríos Alto Purús y Curanja (Tovar, 1998; Tovar et al., 1998), complementándose con un inventario de mamíferos y un estudio de pecaríes (INRENA, 2001), lobos de río y del perro de orejas cortas (ADAR, 2002). Adicionalmente, Aquino (1990), realizó un reconocimiento de las poblaciones de Cacajao calvus "huapo rojo" en la cuenca del río Ucayali, Amazonas y Trapiche.

El presente trabajo documenta la diversidad de mamíferos para la región de Ucayali en base a una revisión de bases de datos de museos nacionales e internacionales y literatura especializada e incluye: 1) Una lista de especies presentes para el área de estudio, señalándose las especies endémicas, raras o listadas en alguna categoría de conservación y 2) Un mapa de los vacíos de información.

Área de Estudio

La región de Ucayali se localiza en la parte centro oriental del Perú, desde $7^{\circ} 21^{\prime} 15^{\prime}$ 'S hasta $11^{\circ} 27^{\prime} S$ y desde 70²9'54”O hasta los 7557'10”O aproximadamente. Tiene una extensión territorial de $102410.55 \mathrm{~km}^{2}$ equivalente al $7.97 \%$ del territorio nacional. Presenta un clima cálido y húmedo con abundantes precipitaciones pluviales y una configuración fisiográfica relacionada directamente con tres pisos naturales: Ceja de Selva, Selva Alta y Selva Baja (Garayar et al., 2005). El río Ucayali, del cual recibe su nombre, es uno de los principales afluentes del gran río Amazonas, tiene su origen en la confluencia de los ríos Urubamba y Tambo y cruza el departamento de sur a norte abarcando casi la totalidad del territorio, exceptuando la provincia del Purús y parte de la provincia de Atalaya, cuyas aguas pertenecen a los ríos Purús y Yurúa, respectivamente.

En Ucayali se encuentran cinco áreas naturales protegidas: Los parques nacionales Cordillera Azul y Alto Purús, las reservas comunales El Sira y Purús y la Zona Reservada Sierra del Divisor. Así también, se presentan numerosas comunidades nativas, bosques de producción permanente y concesiones.

\section{Métodos.}

Se consultaron las bases de datos disponibles de museos internacionales: American Museum Natural History (AMNH); Field Museum of Natural History (FMNH); Museum of Zoology of Louisiana State University (LSUMZ), Museum of Vertebrate Zoology, Berkeley (MVZ), California Academy of Sciences (CAS); y museos nacionales: Museo de Historia Natural, Universidad de San Marcos (MUSM); además de la información de gazeteros electrónicos (www.gbif.net y http://manisnet.org). Se verificó la correcta identificación de los especímenes presentes en la colección del MUSM. Registros de las bases de datos de museos internacionales considerados dudosos o no apoyados por distribución o la literatura no fueron incluidos.

También se incluyó información bibliográfica publicada y no publicada disponible para la región, tales como reportes de inventarios realizados en las Áreas Naturales Protegidas (ANP), descripción de especies en el Oriente Peruano, e información proveniente de estudios en las comunidades indígenas. La nomenclatura y la validez de estos datos también fueron revisados.

Se utilizó el programa Arc View 3.2 para visualizar con mayor precisión los registros presentes en el área de estudio, mapear la distribución real y diferenciar las especies registradas dentro y fuera de las áreas naturales protegidas. Esta información se analiza en contexto con capas digitales de centros poblados, vías de acceso, ríos y ANP; con el fin de observar cuál es la distribución de las especies registradas en Ucayali y los principales agentes antrópicos que pueden afectar la supervivencia de las especies.

Para determinar la lista de especies protegidas se consideraron tres categorías de conservación: la lista roja de la Unión Internacional para la Conservación de la Naturaleza (UICN, 2008), la Legislación Peruana (D.S. 034-2004) y los apéndices de la Convención Internacional sobre el Comercio de Especies Amenazadas de Fauna y Flora (CITES, 2006).

Se sigue la nomenclatura taxonómica empleada por Wilson \& Reeder (2005), Gardner (2008) y Pacheco et al. (2009); excepto por cambios o adiciones recientes incluidas aquí.

\section{Resultados y discusión. \\ Composición de especies}

Se registran aquí 192 especies de mamíferos, en 11 órdenes y 35 familias; representando el 38\% del total de mamíferos registrados para Perú (508 especies, Pacheco et al., 2009), confirmando así la alta diversidad de mamíferos existente en la selva baja (Pacheco, 2002) (Tabla 1). Esta diversidad se divide en tres grupos: el de los mamíferos pequeños (139 especies), mamíferos grandes (51 especies) y mamíferos acuáticos (2 especies). El primer grupo incluye a 86 murciélagos, 36 roedores y 17 marsupiales; y en conjunto fueron el grupo más diverso con el $73 \%$ del total de especies. En comparación, los lagomorfos, perisodáctilos y sirenios son los órdenes menos diversos, cada uno con una sola especie (Figura 1). A nivel de familias, Phyllostomidae y Didelphidae fueron las más diversas con 59 (31\%) y 17 especies (9\%) respectivamente.

Se encontraron 86 localidades diferentes con registros de mamíferos, distribuidas mayormente a lo 


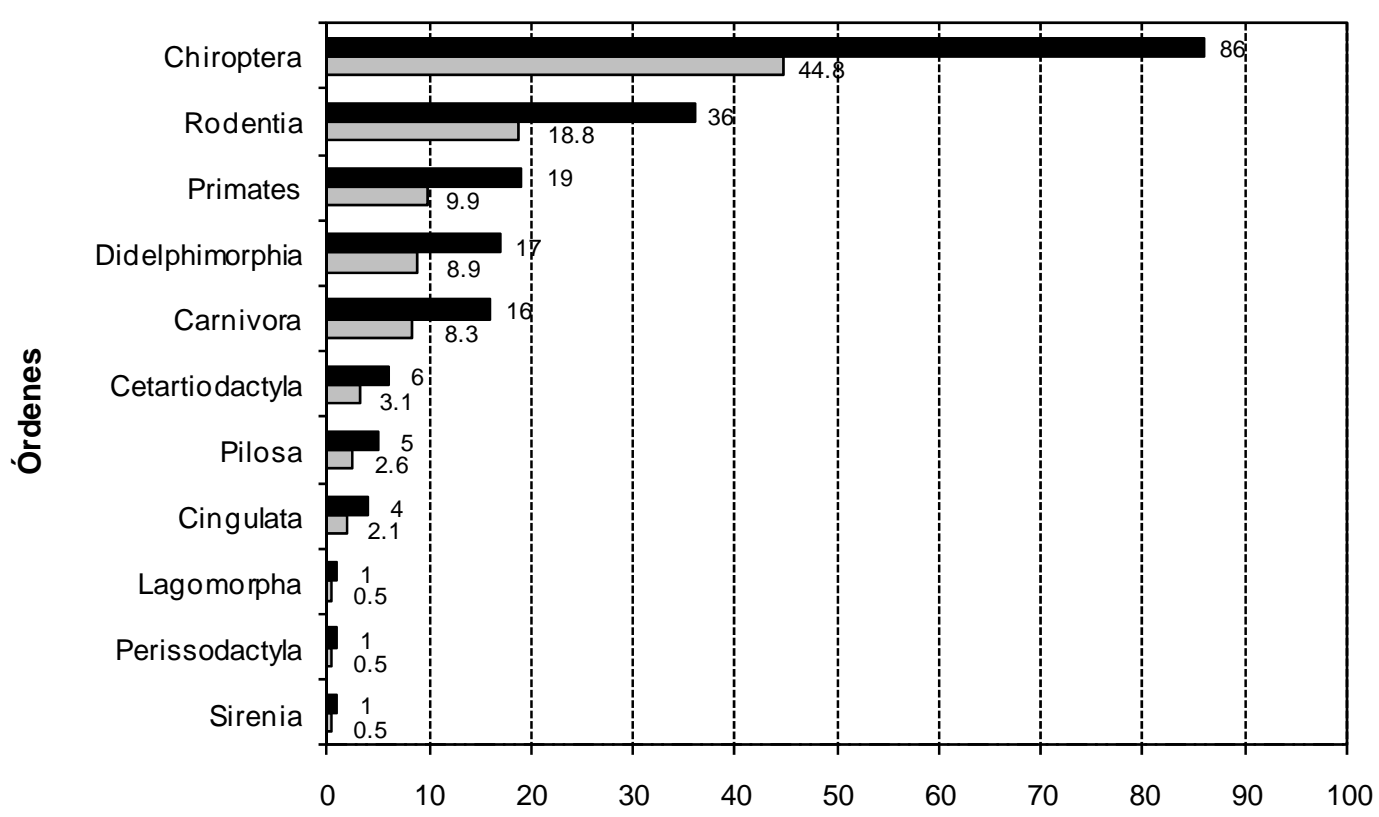

Figura 1. Total de mamíferos por Orden taxonómico registrados en Ucayali, indicando el número de especies y el porcentaje que éstas representan. Barras negras = especies, barras grises $=$ porcentaje.

largo de los principales ríos (Figura 2).

En Ucayali, el $21.4 \%$ del territorio se encuentra protegido por el estado en cinco áreas naturales protegidas. Se contabilizaron 55 especies en tres de ellas: El Parque Nacional Cordillera Azul (10 especies), la Reserva Nacional Sierra del Divisor (39 especies) y la Reserva Comunal Purús (16 especies). Algunas de estas especies están incluidas en más de una zona. En las otras dos áreas protegidas no existen datos confiables. INRENA (2005) lista 194 especies de mamíferos para el Parque Nacional del Alto Purús, pero lamentablemente no incluye la fuente que soporta los registros. La Reserva Comunal El Sira no presenta registro alguno, lo cual se explica por el acceso restringido a la zona, únicamente por vía fluvial.

El 78.6\% restante de Ucayali se encuentra en áreas no protegidas, donde se registran 189 especies; 133 de ellas capturadas sólo en esta área. Esta alta diversidad, mayor a la encontrada en las ANP, es explicable por ser zonas de fácil acceso, lo que ha facilitado una mayor cantidad de expediciones científicas. Nuestros resultados sugieren también que la diversidad no ha sido un factor crítico para el establecimiento de las ANP.

Especies Endémicas y Protegidas

Tres especies del orden Rodentia endémicas para el Perú han sido registradas en Ucayali: la ardilla Sciurus pyrrhinus, la rata acuática Neusticomys peruviensis y la rata espinosa Makalata rhipidura, siendo en la actualidad escasa la información referente a estas especies.
Se registran 52 especies (en 9 órdenes) protegidas por alguna categoría de conservación: 20 especies son consideradas amenazadas por la IUCN, incluyendo a tres En Peligro (EN): Ateles chamek, Lagothrix cana y Pteronura brasiliensis; ocho especies en condición Vulnerable (VU) y nueve especies en situación Casi Amenazada (NT). Unas 17 especies son registradas por la Legislación Peruana, incluyendo a tres En Peligro (EN): Trichechus inunguis, Lontra longicauda y Pteronura brasiliensis; 10 en condición Vulnerable (VU) y cuatro en situación de Casi Amenazado (NT). Finalmente, 44 especies son registradas por CITES, 12 de las cuales se encuentran citadas en el Apéndice I, conformado principalmente por los félidos del Orden Carnivora, 22 en el apéndice II y 10 en el apéndice III (Tabla 1).

De todas las especies protegidas, la nutria grande Pteronura brasiliensis (Carnivora) es considerada la especie más amenazada, categorizada como En Peligro (EN) por IUCN, DS 034 y en el Apéndice I de CITES, seguida por el manatí Trichechus inunguis (Sirenia) y la nutria Lontra longicaudis (Carnivora), ambos catalogados En Peligro (EN) por DS 034 y en el Apéndice I de CITES debido a la cacería intensiva de la que fueron objeto. La protección de estas especies debería ser una prioridad.

Vacíos de Información

En el mapa de Ucayali (Figura 2), se observan dos zonas diferenciadas que no presentan registros de mamíferos, representando Vacíos de Información: 1) la zona Nor Oriental de Ucayali, que pertenece en su 
totalidad a la Reserva Nacional Sierra del Divisor, y 2) la zona oriental de Ucayali, conformada por el territorio cruzado por el río Yucanya (distrito de Masisea), el río Yurúa (distrito de Yurúa) y la cuenca baja del río Purús (que pertenece al Parque Nacional a las concesiones forestales que se encuentran dadas en concesión dentro de los bosques de producción permanente en Ucayali (59.3\%, Portal Agrario, 2009). Estas concesiones, si bien es cierto, son cedidas para poder hacer un buen uso de los recursos y de esta

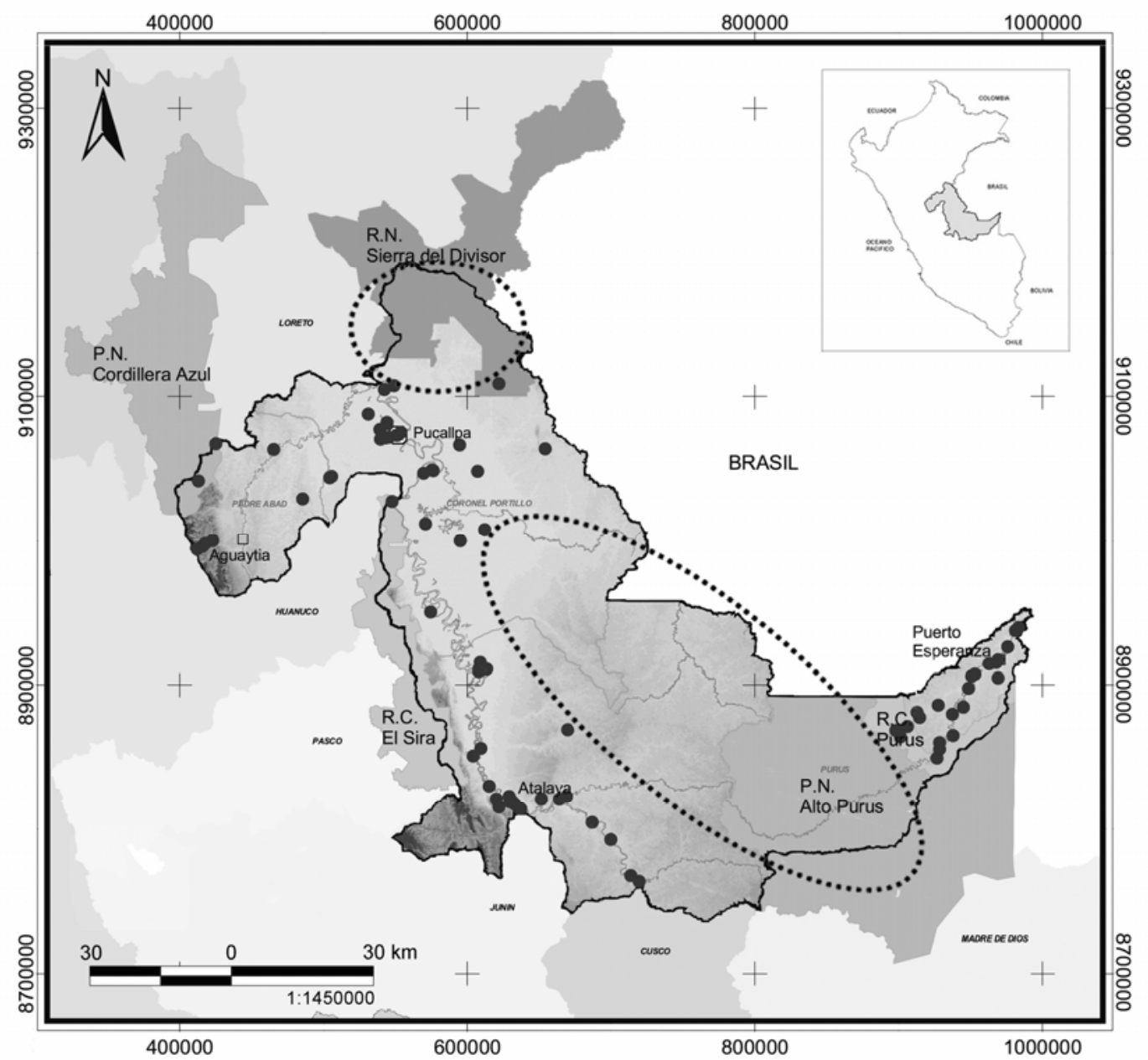

Figura 2. Mapa de Ucayali con las ANP presentes, la ubicación de los registros de especies de mamíferos y los vacíos de información. Puntos negros son localidades con registros de especies.

Alto Purús). Por lo que se sugiere dar prioridad a estas zonas en estudios de diversidad y conservación.

Esta diversidad, aún inexplorada, está sujeta a diversas amenazas. Entre las más serias se encuentran: la actividad maderera, la extracción de productos del bosque, la falta de implementación en el manejo y control de las áreas protegidas, el contacto con grupos indígenas en aislamiento, la migración de otras poblaciones humanas a la región; así como los proyectos de construcción de carreteras. Si bien en la actualidad la magnitud de estas amenazas no es significativa debido a las condiciones de aislamiento de la región, se observa una tendencia creciente al aumento de las mismas, sobre todo en lo concerniente a la tala ilegal de madera. Las actividades mencionadas previamente, se ven potenciadas debido manera ejercer un uso sostenible de los mismos, en la actualidad, algunas de ellas tienen poca o casi nula fiscalización, lo cual impide tener un conocimiento real de cuál es la dinámica dentro de ellas y por ende de sus recursos.

\section{Conclusiones.}

Ucayali es uno de los departamentos con mayor diversidad de mamíferos en el Perú con un significativo número de especies endémicas y amenazadas. Los dos Vacíos de Información encontrados indican que una gran parte de su territorio está aún inexplorada, sugiriendo que la diversidad real de Ucayali es mucho mayor a la aquí registrada. Igualmente, la diversidad de las ANP es pobremente conocida; sin duda un mayor conocimiento de su 
diversidad permitirá un mejor soporte para la valoración y conservación de las mismas.

\section{Agradecimientos.}

A los miembros del departamento de Mastozoología del Museo de Historia Natural por el apoyo en la identificación de los especímenes, a World Wildlife Fund Perú (WWF Perú) por el financiamiento parcial para esta investigación y al Mg. Jose Luis Mena por promover la presente publicación.

\section{Literatura citada.}

ADAR. 2002. Evaluación de la fauna bajo presión de caza en los ríos Purús y Curanja, Ucayali, Perú. Reporte de la Cuarta Fase del Proyecto de Evaluación y Asesoría Medio Ambiental en el Río Purús.

Aniskin M., Varshavskii A.A., Isaef S.I. \& Malyugin V.M. 1991. Comparative analysis of differentially G- and Cstained chromosomes of five species of family Didelphidae (Marsupialia). Genetika. 27:504-514.

Aquino R. 1990. Reconocimiento preliminar de poblaciones de Cacajao calvus "Huapo rojo" (Cebidae, Primates) en el Oriente Peruano. En La Primatología en el Perú. Investigaciones Primatológicas (1973-1985). Proyecto peruano de primatología "Manuel Moro Sommo". DGFF-IVITA-OPS/OMS.

Aquino R. \& Encarnación F. 1994. Los primates del Perú. Primate Report. 40: 43-129.

Aquino R., Alvarez J. \& Mulanovich A. 2005. Diversidad y estado de conservación de primates en las Sierras de Contamana, Amazonía Peruana. Revista Peruana de Biología. 12(3): 427-434.

Bartlett E. 1871. Notes on the monkeys of eastern Peru. Proceedings of the Zoological Society of London. 1871: 217-220, pl. XIII.

Convention on International Trade in Endangered Species of Wild Fauna and Flora. CITES 2006. Apéndices I, II y III. http://www.cites.org/eng /app/appendices.html> Consultado el 15 de Noviembre de 2008.

Conservation of Migratory Species (CMS). 2007. Whales \& Dolphins, Review of small cetaceans: Distribution, Behaviour, Migration and Threats by Boris M. Culik (Kiel, Germany) 2003. http://www.cms.int/reports/small_cetaceans/data/S_flu viatilis/s_fluviatilis.htm. Consultado el 10 de Julio del 2007.

Davenport L. 2003. La Problemática de las Aguas: los lobos de río y los hábitats acuáticos de la Zona Reservada del Alto Purús. Páginas 125-135. En: R. Leite Pitman, N. Pitman y P. Alvarez (eds). Alto Purús: Biodiversidad, Conservación y Manejo. Center for Tropical Conservation.

Decreto Supremo (D.S.) 034-2004-AG. Aprueban categorización de especies amenazadas de fauna silvestre y prohíben su caza, captura, tenencia, transporte o exportación con fines comerciales. El Peruano, 2004: 276853-276855.

Dorst J. 1953. Étude d'une collection de chiroptères du Rio Ucayali, Pérou. Bulletin du Muséum national d'Histoire naturelle. 25: 269-271.

Emmons L.H. \& Feer F. 1990. "Neotropical Rainforest Mammals: A Field Guide". Universtity of Chicago Press, Chicago.
Emmons L.H. \& Feer F. 1997. Neotropical Rainforest Mammals. A Field Guide. 2da Edición. University of Chicago Press, Chicago.

Fooden J. 1963. A Revision of the Woolly Monkeys (Genus Lagothrix). Journal of Mammalogy. 44(2): 213-247.

Garayar C., Vallenas H. \& Coronado G. 2005. Gran Atlas del Perú. Ediciones Peisa S. S.C. Lima.

Gardner A.L. 2008. Mammals of South America, Volume 1. Marsupials, Xenarthrans, Shrews, and Bats. The University of Chicago Press, Chicago.

Gervais P. 1855. Recherches sur les mammifères fossiles de l'Amérique du Sud. En: F. de Castelnau: Expédition dans les parties centrales de l'Amérique du Sud, de Rio de Janeiro à Lima, et de Lima au Para; exécuté par ordre du Goubernenment français pendant les années 1843 à 1847 sous la direction du comte Francis de Castelnau. Zoologie. 7: 1-63.

Gil Navarro J.G. 2004. Aprovechamiento de la Fauna Silvestre en comunidades Cashinahua del río Curanja y Purús Informe Técnico I. Con la colaboración de: Fundación Gordon \& Betty Moore y WWF-Holanda.

Hershkovitz P. 1988. Origin, speciation, and distribution of South American titi monkeys, genus Callicebus (family Cebidae, Platyrrhini). Proceedings of the Academy of Natural Sciences of Philadelphia. 140(1): 240-272.

Hood C. \& Gardner A.L. 2007. Family Emballonuridae, Páginas 188-207. En: Gardner A. (editor.) Mammals of South America, Volume 1. Marsupials, Xenarthrans, Shrews, and Bats. The University of Chicago Press, Chicago.

Husar S.L. 1977. Trichechus inunguis. Mammalian Species. 72: $1-4$.

INRENA. 2001. Primera evaluación poblacional de pecaries en la zona de alto Purus-Ucayali. Dirección General Forestal y de Fauna Silvestre. Lima-Perú.

2005. Parque Nacional Alto Purús Plan Maestro 2005-2010. Proyecto INRENA/GEF-BM Instituto de Recursos Naturales-INRENA. Lima.

IUCN. 2008. Red List of Threatened Species. $<$ www.redlist.org > . Consultado en Febrero del 2009.

Leite R., Beck H. \& Velazco P. 2003. Mamíferos terrestres y arbóreos de la selva baja de la amazonía peruana: entre los ríos Manu y Alto Purús. Páginas 109-122. En: Leite R., Pitman N. \& Álvarez P.; (editores.) Alto Purús: Biodiversidad Conservación y Manejo Center for Tropical Conservation. Perú, Lima.

Marques-Aguiar S.A. 2007. Genus Artibeus Leach, 1821. Páginas 301-321 En: Gardner A.L. (editor) Mammals of South America. Volume I: Marsupials, Xenarthrans, Shrews, and Bats. University of Chicago Press, Chicago.

Pacheco V. \& Arias L. 2001. Mamíferos. Páginas 85-88, 226-227. En: W.S. Alverson, L.O. Rodriguez, y D.K. Moskovits (editores) Perú: Biabo Cordillera Azul. The Field Museum, Chicago.

Pacheco V. 2002. Mamíferos del Perú. Páginas 503-549. En: G. Ceballos y J. Simonetti (editores) Diversidad y Conservación de los Mamíferos Neotropicales CONABIO-UNAM. México, D.F.

Pacheco V., Cadenillas R., Salas E., Tello C. \& Zeballos H. 2009. Diversidad y endemismo de los mamíferos del Perú. Revista Peruana de Biología. 16(1): 5-32.

Percequillo A.R., Carmignotto A.P. \& de J. Silva M.J. 2005. A new species of Neusticomys (Ichthyomyini, 
Sigmodontinae) from Central Brazilian Amazonia. Journal of Mammalogy. 86(5): 873-880.

Portal Agrario. 2009. Estado de la Situación Forestal en el Perú.

http://www.portalagrario.com.pe/rrnn/rrnn_f_anexo.sht ml. Consultado el 15 de julio del 2009.

Reig O.A., Gardner A.L., Bianchi N.O. \& Patton J.L. 1977. The chromosomes of the didelphidae (Marsupialia) and their evolutionary significance. Biological Journal of the Linnean Society. 9: 191-216.

Sanborn C.C. 1949. Mammals from the Rio Ucayali, Peru. Journal of Mammalogy. 30(3): 277-288.

Thomas O. 1924. New South American mammals. Annals and Magazine of Natural History, Series. 9 13: 234237.

Thomas O. 1928. The Godman-Thomas expedition to Peru.-
VII. On mammal obtained by Mr. Hendee at Pebas and Iquitos, Upper Amazons. Annals and Magazine of Natural History. 2: 285-294.

Tovar N.A. 1998. Vale un Purús: Bosques, Ríos y peruanidad. Despertar Pozucino. 9:51-54.

Tovar N.A., Nuñez G. \& Chang J. 1998. Informe de viaje: Río Alto Purús, Ucayali, Perú (Preliminar). Informe inédito.

Voss R.S. \& Emmons L.H. 1996. Mammalian diversity in neotropical lowland rainforests: A preliminary assessment. Bulletin of the American Museum of Natural History. 230: 1-115.

Wilson D.E. \& Reeder D.M. Eds. 2005. Mammal Species of the World: A Taxonomic and Geographic Reference, 3rd ed. Johns Hopkins University Press, Baltimore, Maryland.

Tabla 1. Lista de las especies de mamíferos registrados en Ucayali, indicando su presencia en las ANP y su categoría de amenaza.

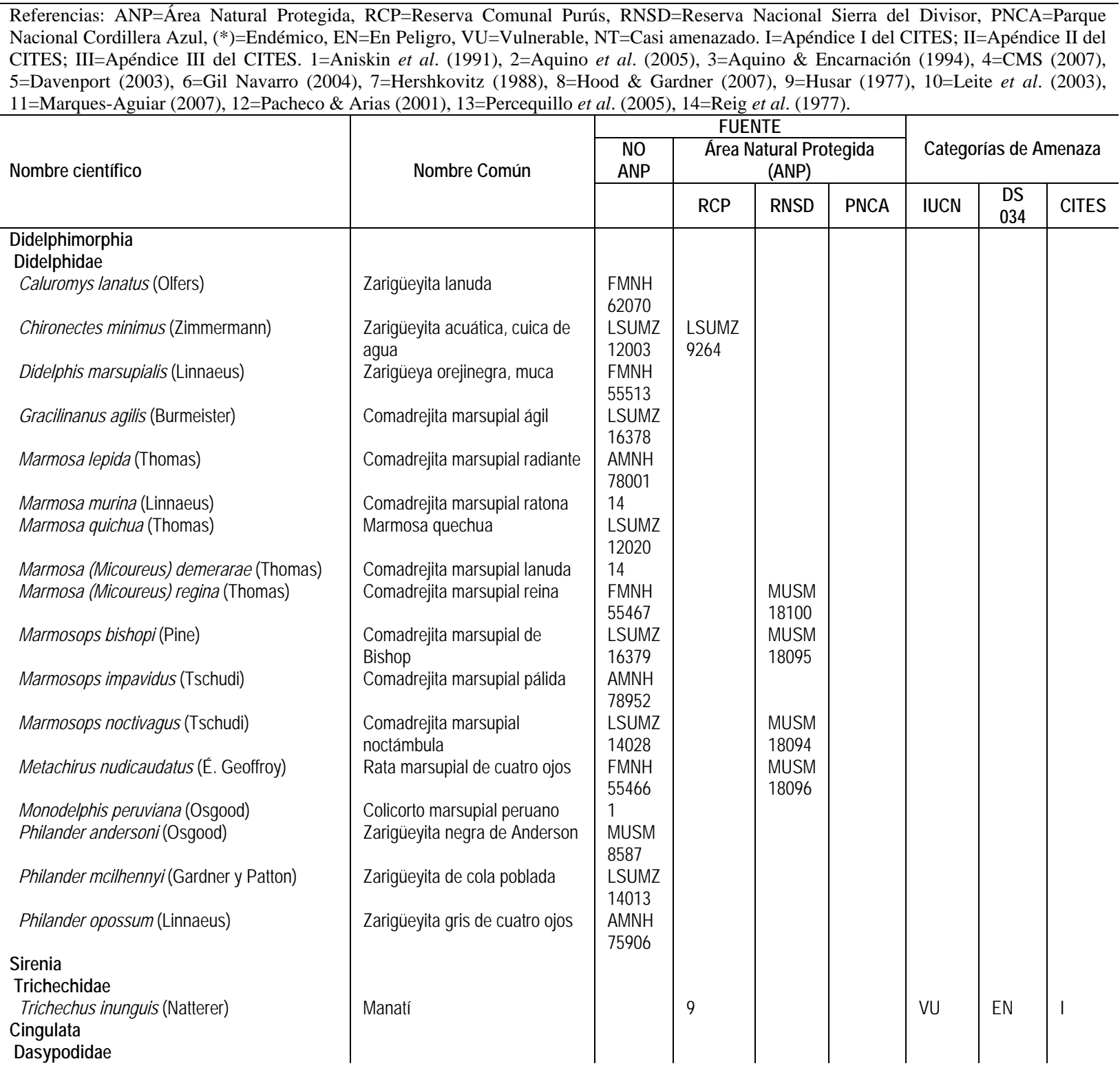


Tabla 1. Lista de las especies de mamíferos registrados en Ucayali, indicando su presencia en las ANP y su categoría de amenaza.

Referencias: ANP=Área Natural Protegida, RCP=Reserva Comunal Purús, RNSD=Reserva Nacional Sierra del Divisor, PNCA=Parque Nacional Cordillera Azul, $\left({ }^{*}\right)=$ Endémico, EN=En Peligro, VU=Vulnerable, NT=Casi amenazado. I=Apéndice I del CITES; II=Apéndice II del CITES; III=Apéndice III del CITES. 1=Aniskin et al. (1991), 2=Aquino et al. (2005), 3=Aquino \& Encarnación (1994), 4=CMS (2007), 5=Davenport (2003), 6=Gil Navarro (2004), 7=Hershkovitz (1988), 8=Hood \& Gardner (2007), 9=Husar (1977), 10=Leite et al. (2003), 11=Marques-Aguiar (2007), 12=Pacheco \& Arias (2001), 13=Percequillo et al. (2005), 14=Reig et al. (1977).

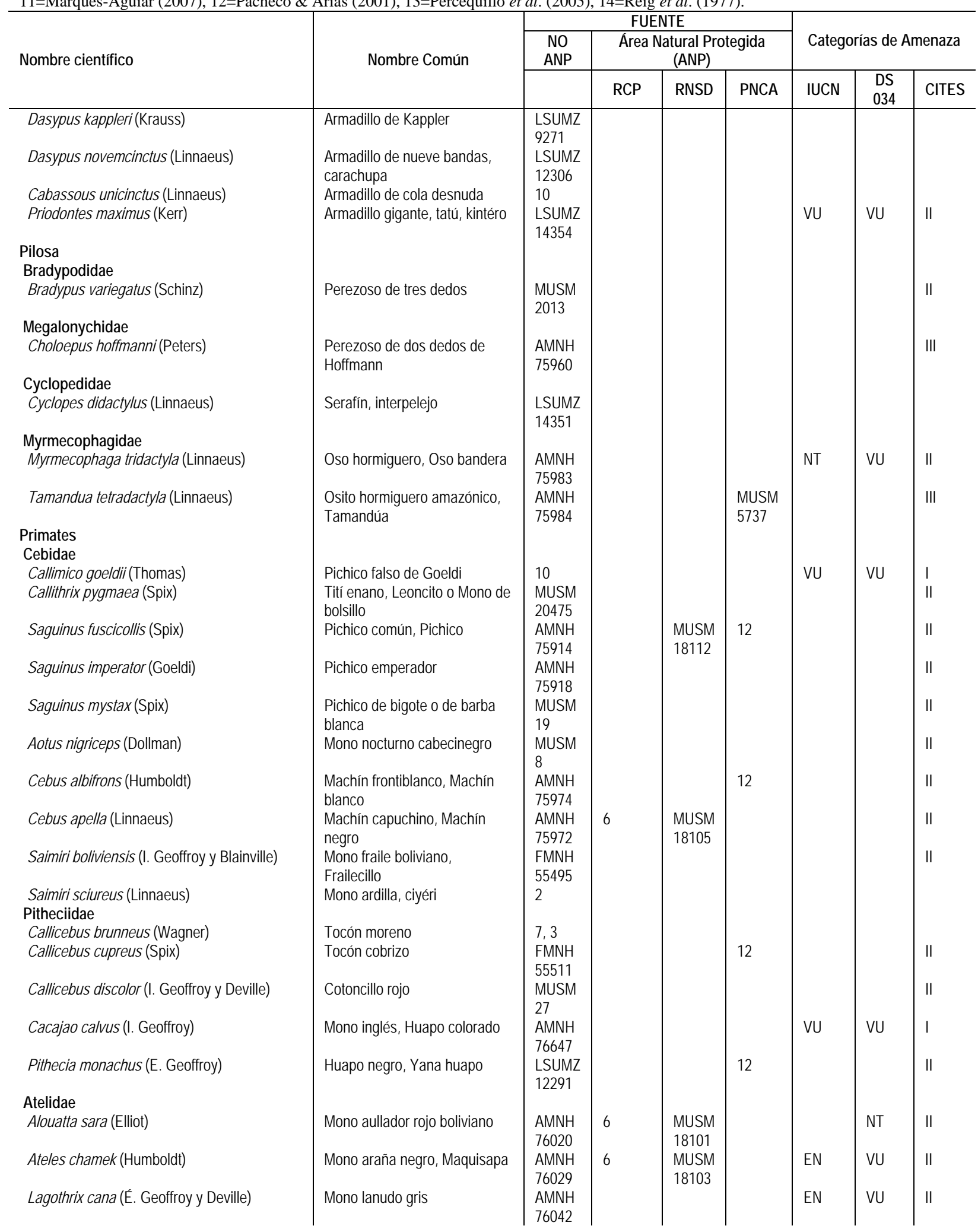


Tabla 1. Lista de las especies de mamíferos registrados en Ucayali, indicando su presencia en las ANP y su categoría de amenaza. Referencias: ANP=Área Natural Protegida, RCP=Reserva Comunal Purús, RNSD=Reserva Nacional Sierra del Divisor, PNCA=Parque
Nacional Cordillera Azul, ( ${ }^{*}$ =Endémico, EN=En Peligro, VU=Vulnerable, NT=Casi amenazado. I=Apéndice I del CITES; II=Apéndice II del
CITES; III=Apéndice III del CITES. 1=Aniskin et al. (1991), 2=Aquino et al. (2005), 3=Aquino \& Encarnación (1994), 4=CMS (2007),
5=Davenport (2003), 6=Gil Navarro (2004), 7=Hershkovitz (1988), 8=Hood \& Gardner (2007), 9=Husar (1977), 10=Leite et al. (2003),
11=Marques-Aguiar (2007), 12=Pacheco \& Arias (2001), 13=Percequillo et al. (2005), 14=Reig et al. (1977).

\begin{tabular}{|c|c|c|c|c|c|c|c|c|}
\hline \multirow{3}{*}{ Nombre científico } & \multirow{3}{*}{ Nombre Común } & \multicolumn{4}{|c|}{ FUENTE } & \multirow{2}{*}{\multicolumn{3}{|c|}{ Categorías de Amenaza }} \\
\hline & & \multirow[t]{2}{*}{$\begin{array}{l}\text { NO } \\
\text { ANP }\end{array}$} & \multicolumn{3}{|c|}{$\begin{array}{l}\text { Área Natural Protegida } \\
\text { (ANP) }\end{array}$} & & & \\
\hline & & & RCP & RNSD & PNCA & IUCN & $\begin{array}{c}\text { DS } \\
034\end{array}$ & CITES \\
\hline Lagothrix poeppigii (Schinz) & Mono lanudo de Pöppig & $\begin{array}{l}\text { MUSM } \\
18108\end{array}$ & & & & $\mathrm{VU}$ & NT & II \\
\hline $\begin{array}{l}\text { Rodentia } \\
\text { Sciuridae }\end{array}$ & & & & & & & & \\
\hline Microsciurus flaviventer (Gray) & Ardillita de vientre amarillo & $\begin{array}{l}\text { AMNH } \\
78942\end{array}$ & & $\begin{array}{l}\text { MUSM } \\
18116\end{array}$ & & & & \\
\hline Sciurus ignitus (Gray) & Ardilla ígnea & $\begin{array}{l}\text { AMNH } \\
78943\end{array}$ & & & & & & \\
\hline Sciurus igniventris (Wagner) & Ardilla de vientre rojo & $\begin{array}{l}\text { AMNH } \\
75922\end{array}$ & & & & & & \\
\hline Sciurus pyrrhinus* (Thomas) & Ardilla rojiza & $\begin{array}{l}\text { FMNH } \\
64296\end{array}$ & & & & & $\mathrm{VU}$ & \\
\hline Sciurus spadiceus (Olfers) & Ardilla baya & $\begin{array}{l}\text { FMNH } \\
55488\end{array}$ & & $\begin{array}{l}\text { MUSM } \\
18159\end{array}$ & & & & \\
\hline Cricetidae & & & & & & & & \\
\hline Euryoryzomys macconnelli (Thomas) & Ratón arrozalero de Macconel & $\begin{array}{l}\text { LSUMZ } \\
14366\end{array}$ & & $\begin{array}{l}\text { MUSM } \\
18118\end{array}$ & & & & \\
\hline Euryoryzomys nitidus (Thomas) & Ratón arrozalero lustroso & $\begin{array}{l}\text { LSUMZ } \\
12333\end{array}$ & & & & & & \\
\hline Holochilus sciureus (Wagner) & Rata ardilla de pantano & $\begin{array}{l}\text { FMNH } \\
55471\end{array}$ & & & & & & \\
\hline Hylaemays perenensis (J. A. Allen) & Ratón arrozalero cabezudo & $\begin{array}{l}\text { LSUMZ } \\
12348\end{array}$ & & $\begin{array}{l}\text { MUSM } \\
18136\end{array}$ & & & & \\
\hline Hylaemays yunganus (Thomas) & $\begin{array}{l}\text { Ratón arrozalero de las } \\
\text { Yungas }\end{array}$ & $\begin{array}{l}\text { MVZ } \\
136585\end{array}$ & & & & & & \\
\hline Neacomys spinosus (Thomas) & Ratón espinoso común & $\begin{array}{l}\text { LSUMZ } \\
12386\end{array}$ & & & & & & \\
\hline Nectomys apicalis (Peters) & Rata de agua ecuatoriana & $\begin{array}{l}\text { LSUMZ } \\
16708\end{array}$ & & & & & & \\
\hline $\begin{array}{l}\text { Neusticomys peruviensis* (Musser y } \\
\text { Gardner) }\end{array}$ & $\begin{array}{l}\text { Nectomys de la amazonía } \\
\text { tropical }\end{array}$ & $\begin{array}{l}\text { LSUMZ } \\
14407\end{array}$ & 13 & & & & VU & \\
\hline Oecomys bicolor (Tomes) & Ratón arrozalero bicolor & $\begin{array}{l}\text { FMNH } \\
64298\end{array}$ & & & & & & \\
\hline Oecomys superans (Thomas) & Ratón arrozalero selvático & $\begin{array}{l}\text { LSUMZ } \\
12331\end{array}$ & & & & & & \\
\hline Oecomys trinitatis (J. A. Allen y Chapman) & Ratón arrozalero peludo & $\begin{array}{l}\text { LSUMZ } \\
12330\end{array}$ & & & & & & \\
\hline Oligoryzomys microtis (J. A. Allen) & $\begin{array}{l}\text { Ratón arrozalero de oreja } \\
\text { pequeña }\end{array}$ & $\begin{array}{l}\text { AMNH } \\
76105\end{array}$ & & & & & & \\
\hline Scolomys ucayalensis (Pacheco) & Ratón espinoso del Ucayali & & & $\begin{array}{l}\text { MUSM } \\
18160\end{array}$ & & & & \\
\hline Erethizontidae & & & & & & & & \\
\hline Coendou bicolor (Tschudi) & Puerco espín arborícola, Erizo & $\begin{array}{l}\text { LSUMZ } \\
16745\end{array}$ & & & & & & \\
\hline Dinomyidae & & & & & & & & \\
\hline Dinomys branickii (Peters) & $\begin{array}{l}\text { Machetero, Pacarana, Picuru } \\
\text { maman }\end{array}$ & $\begin{array}{l}\text { LSUMZ } \\
12320\end{array}$ & & & & VU & & \\
\hline Caviidae & & & & & & & & \\
\hline Hydrochoerus hydrochaeris (Linnaeus) & Ronsoco, Ivéto & $\begin{array}{l}\text { LSUMZ } \\
12463\end{array}$ & 6 & & & & & \\
\hline Dasyproctidae & & & & & & & & \\
\hline Dasyprocta fuliginosa (Wagler) & Añuje, Chapana & $\begin{array}{l}\text { AMNH } \\
76617\end{array}$ & & $\begin{array}{l}\text { MUSM } \\
18115\end{array}$ & & & & \\
\hline Dasyprocta variegata (Tschudi) & Añuje, Cutpe, Agutí & $\begin{array}{l}\text { AMNH } \\
75961\end{array}$ & 6 & & & & & III \\
\hline Myoprocta pratti (Pocock) & Punchana, Añuje menor & $\begin{array}{l}\text { AMNH } \\
75965\end{array}$ & & & & & & \\
\hline $\begin{array}{l}\text { Cuniculidae } \\
\text { Cuniculus paca (Linnaeus) }\end{array}$ & Majaz, Picuro, Zamaño, Liebre & $\begin{array}{l}\text { AMNH } \\
75958\end{array}$ & 6 & $\begin{array}{l}\text { MUSM } \\
18114\end{array}$ & & & VU & III \\
\hline
\end{tabular}


Tabla 1. Lista de las especies de mamíferos registrados en Ucayali, indicando su presencia en las ANP y su categoría de amenaza.

Referencias: ANP=Área Natural Protegida, RCP=Reserva Comunal Purús, RNSD=Reserva Nacional Sierra del Divisor, PNCA=Parque Nacional Cordillera Azul, $\left({ }^{*}\right)=$ Endémico, EN=En Peligro, VU=Vulnerable, NT=Casi amenazado. I=Apéndice I del CITES; II=Apéndice II del CITES; III=Apéndice III del CITES. 1=Aniskin et al. (1991), 2=Aquino et al. (2005), 3=Aquino \& Encarnación (1994), 4=CMS (2007), 5=Davenport (2003), 6=Gil Navarro (2004), 7=Hershkovitz (1988), 8=Hood \& Gardner (2007), 9=Husar (1977), 10=Leite et al. (2003), 11=Marques-Aguiar (2007), 12=Pacheco \& Arias (2001), 13=Percequillo et al. (2005), 14=Reig et al. (1977).

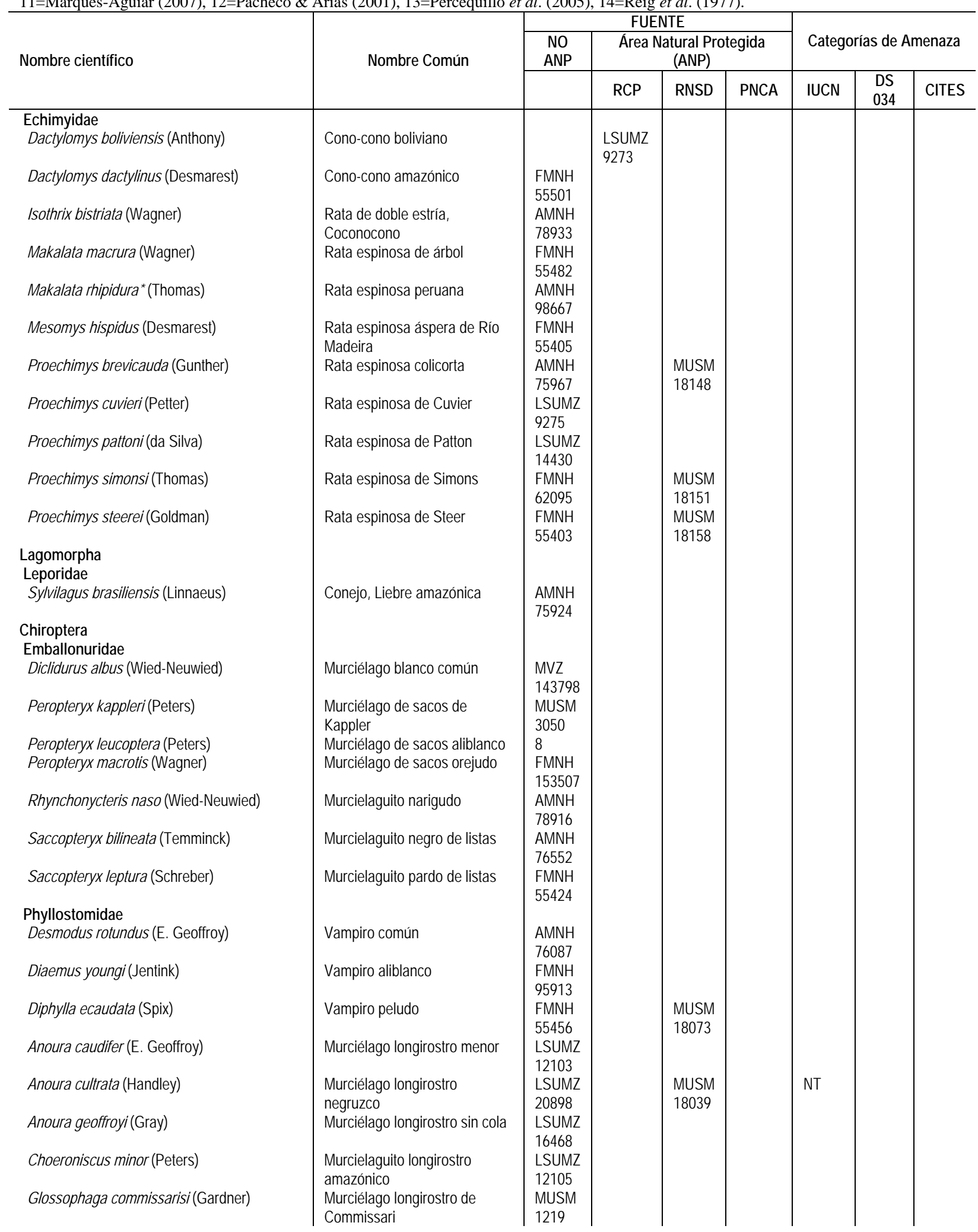


Julio - Diciembre 2009

Tabla 1. Lista de las especies de mamíferos registrados en Ucayali, indicando su presencia en las ANP y su categoría de amenaza.

Referencias: ANP=Área Natural Protegida, RCP=Reserva Comunal Purús, RNSD=Reserva Nacional Sierra del Divisor, PNCA=Parque Nacional Cordillera Azul, $(*)=E n d e ́ m i c o$, EN=En Peligro, VU=Vulnerable, NT=Casi amenazado. I=Apéndice I del CITES; II=Apéndice II del CITES; III=Apéndice III del CITES. 1=Aniskin et al. (1991), 2=Aquino et al. (2005), 3=Aquino \& Encarnación (1994), 4=CMS (2007), 5=Davenport (2003), 6=Gil Navarro (2004), 7=Hershkovitz (1988), 8=Hood \& Gardner (2007), 9=Husar (1977), 10=Leite et al. (2003), 11=Marques-Aguiar (2007), 12=Pacheco \& Arias (2001), 13=Percequillo et al. (2005), 14=Reig et al. (1977).

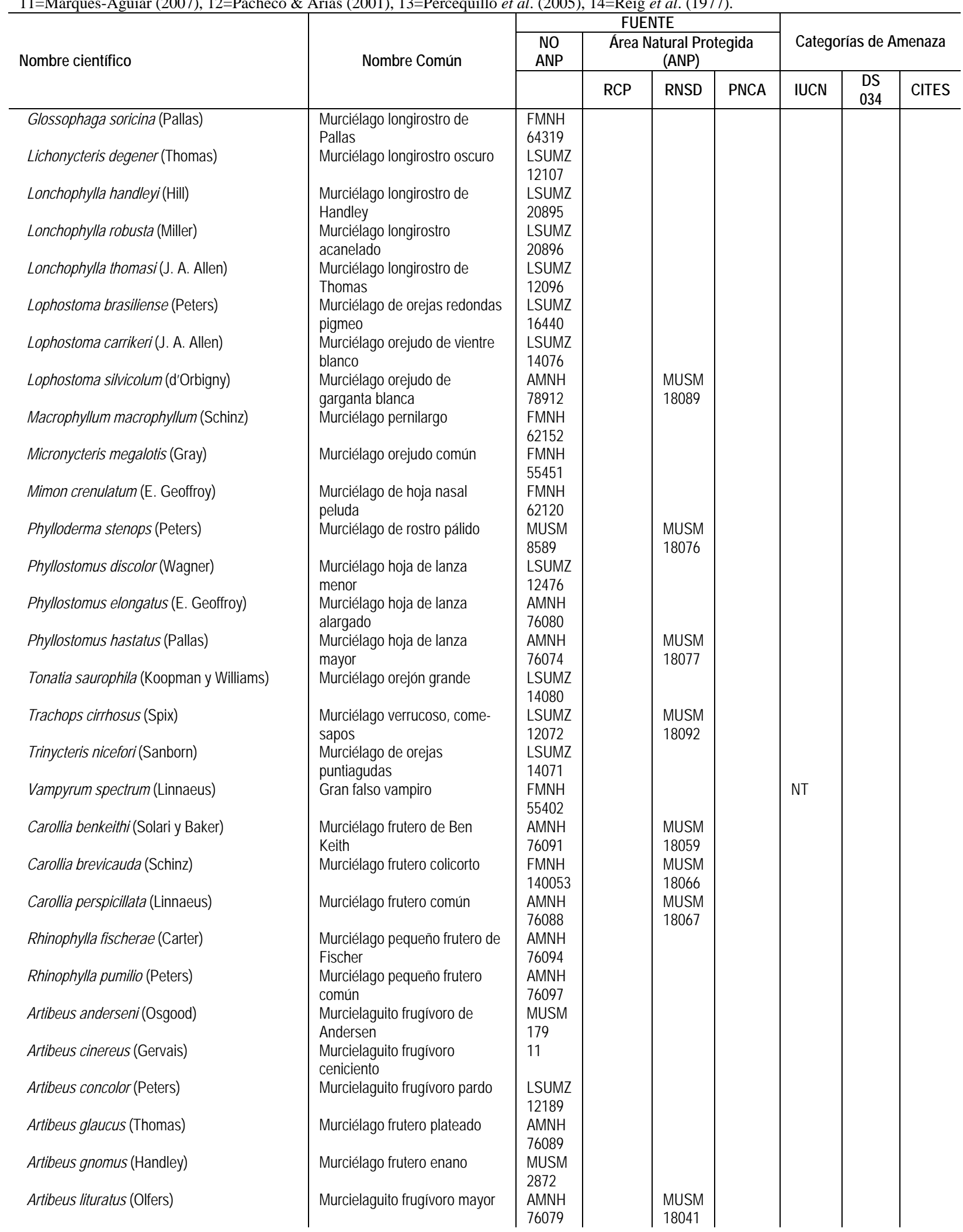


Tabla 1. Lista de las especies de mamíferos registrados en Ucayali, indicando su presencia en las ANP y su categoría de amenaza.

Referencias: ANP=Área Natural Protegida, RCP=Reserva Comunal Purús, RNSD=Reserva Nacional Sierra del Divisor, PNCA=Parque Nacional Cordillera Azul, $\left({ }^{*}\right)=$ Endémico, EN=En Peligro, VU=Vulnerable, NT=Casi amenazado. I=Apéndice I del CITES; II=Apéndice II del CITES; III=Apéndice III del CITES. 1=Aniskin et al. (1991), 2=Aquino et al. (2005), 3=Aquino \& Encarnación (1994), 4=CMS (2007), 5=Davenport (2003), 6=Gil Navarro (2004), 7=Hershkovitz (1988), 8=Hood \& Gardner (2007), 9=Husar (1977), 10=Leite et al. (2003), 11=Marques-Aguiar (2007), 12=Pacheco \& Arias (2001), 13=Percequillo et al. (2005), 14=Reig et al. (1977).

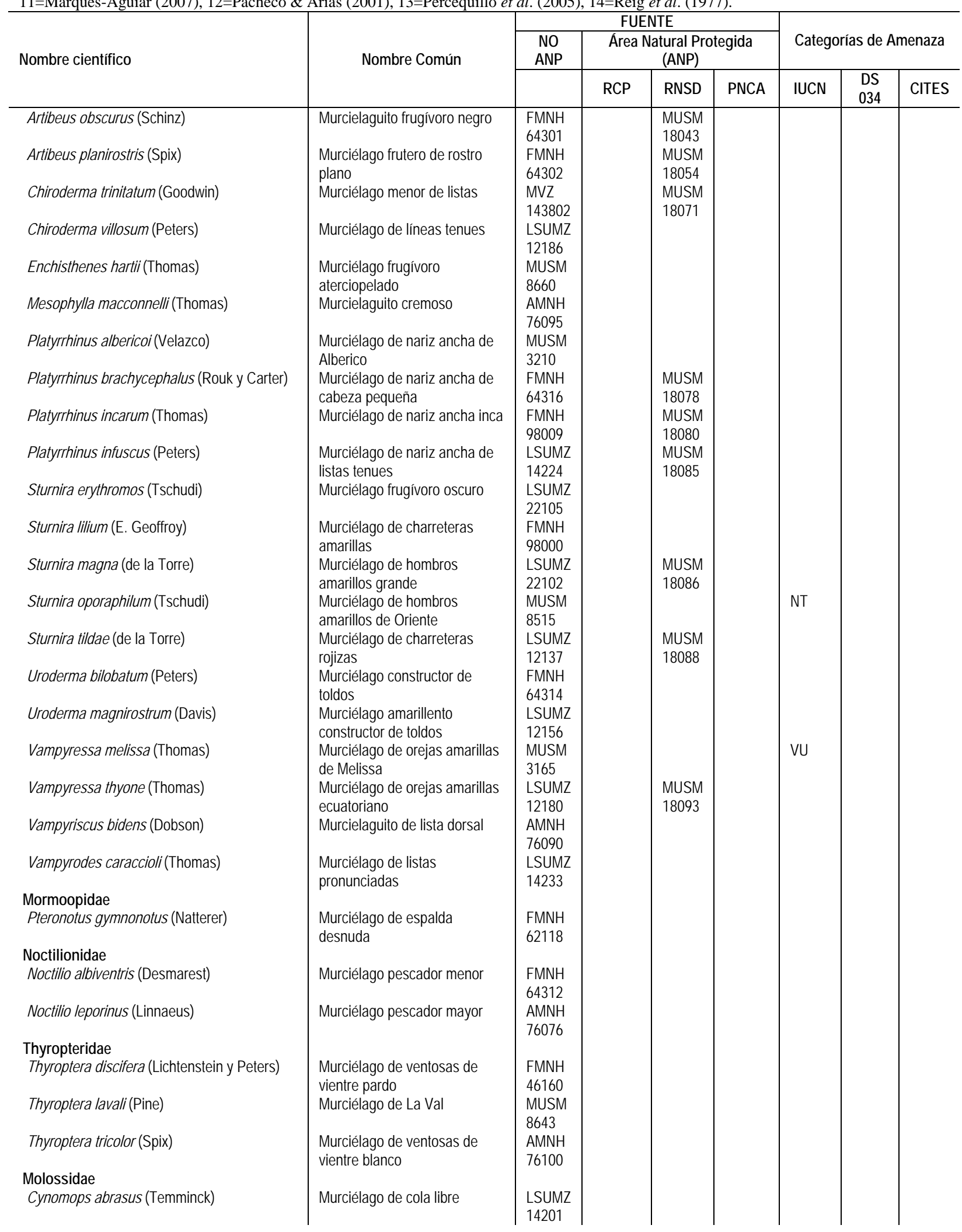


Tabla 1. Lista de las especies de mamíferos registrados en Ucayali, indicando su presencia en las ANP y su categoría de amenaza. Referencias: ANP=Área Natural Protegida, RCP=Reserva Comunal Purús, RNSD=Reserva Nacional Sierra del Divisor, PNCA=Parque
Nacional Cordillera Azul, ( ${ }^{*}$ =Endémico, EN=En Peligro, VU=Vulnerable, NT=Casi amenazado. I=Apéndice I del CITES; II=Apéndice II del
CITES; III=Apéndice III del CITES. 1=Aniskin et al. (1991), 2=Aquino et al. (2005), 3=Aquino \& Encarnación (1994), 4=CMS (2007),
5=Davenport (2003), 6=Gil Navarro (2004), 7=Hershkovitz (1988), 8=Hood \& Gardner (2007), 9=Husar (1977), 10=Leite et al. (2003),
11=Marques-Aguiar (2007), 12=Pacheco \& Arias (2001), 13=Percequillo et al. (2005), 14=Reig et al. (1977).

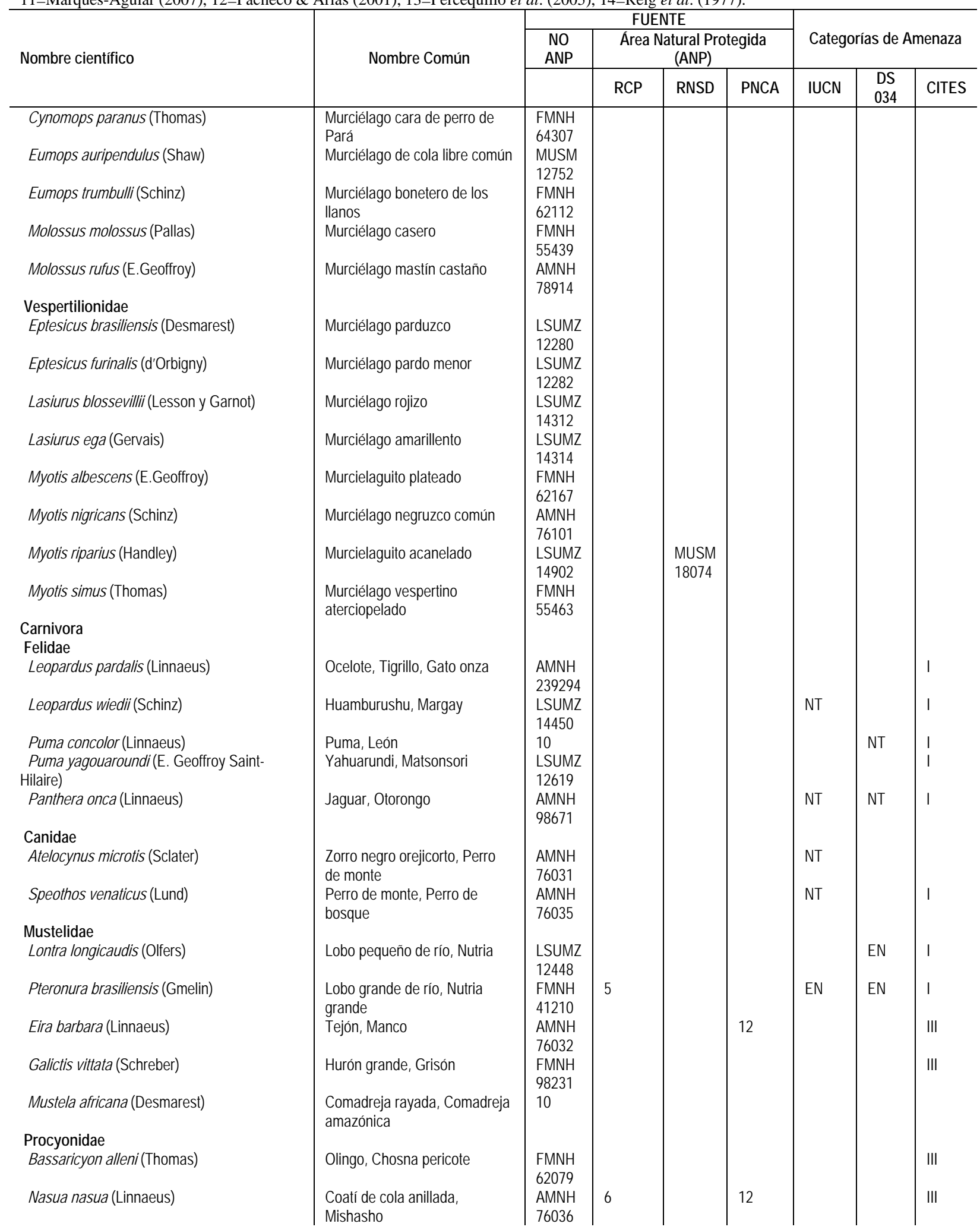


Tabla 1. Lista de las especies de mamíferos registrados en Ucayali, indicando su presencia en las ANP y su categoría de amenaza.

\begin{tabular}{|c|c|c|c|c|c|c|c|c|}
\hline \multicolumn{9}{|c|}{$\begin{array}{l}\text { Referencias: ANP=Área Natural Protegida, RCP=Reserva Comunal Purús, RNSD=Reserva Nacional Sierra del Divisor, PNCA=Parque } \\
\text { Nacional Cordillera Azul, (*)=Endémico, EN=En Peligro, VU=Vulnerable, NT=Casi amenazado. I=Apéndice I del CITES; II=Apéndice II del } \\
\text { CITES; III=Apéndice III del CITES. 1=Aniskin et al. (1991), 2=Aquino et al. (2005), 3=Aquino \& Encarnación (1994), 4=CMS (2007), } \\
\text { 5=Davenport (2003), 6=Gil Navarro (2004), 7=Hershkovitz (1988), 8=Hood \& Gardner (2007), 9=Husar (1977), 10=Leite et al. (2003), } \\
\text { 11=Marques-Aguiar (2007), 12=Pacheco \& Arias (2001), 13=Percequillo et al. (2005), 14=Reig et al. (1977). }\end{array}$} \\
\hline \multirow{3}{*}{ Nombre científico } & \multirow{3}{*}{ Nombre Común } & \multicolumn{4}{|c|}{ FUENTE } & \multirow{2}{*}{\multicolumn{3}{|c|}{ Categorías de Amenaza }} \\
\hline & & \multirow[t]{2}{*}{$\begin{array}{l}\text { NO } \\
\text { ANP }\end{array}$} & \multicolumn{3}{|c|}{$\begin{array}{l}\text { Área Natural Protegida } \\
\text { (ANP) }\end{array}$} & & & \\
\hline & & & $\mathrm{RCP}$ & RNSD & PNCA & IUCN & $\begin{array}{l}\text { DS } \\
034\end{array}$ & CITES \\
\hline Potos flavus (Schreber) & Chosna, Cuchumli & $\begin{array}{l}\text { FMNH } \\
55503\end{array}$ & & & & & & III \\
\hline Procyon cancrivorus (G. [Baron] Cuvier) & Osito cangrejero, Osito lavador & $\begin{array}{l}\text { LSUMZ } \\
14443\end{array}$ & & & & & & \\
\hline $\begin{array}{l}\text { Perissodactyla } \\
\text { Tapiridae }\end{array}$ & & & & & & & & \\
\hline Tapirus terrestris (Linnaeus) & $\begin{array}{l}\text { Tapir del llano amazónico, } \\
\text { Sachavaca }\end{array}$ & $\begin{array}{l}\text { FMNH } \\
55516\end{array}$ & 6 & & 12 & VU & VU & II \\
\hline $\begin{array}{l}\text { Cetartiodactyla } \\
\text { Tayassuidae }\end{array}$ & & & & & & & & \\
\hline Pecari tajacu (Linnaeus) & Sajino & $\begin{array}{l}\text { AMNH } \\
76807\end{array}$ & 6 & $\begin{array}{l}\text { MUSM } \\
18038\end{array}$ & 12 & & & II \\
\hline Tayassu pecari (Link) & Pecarí boquiblanco, Huangana & $\begin{array}{l}\text { FMNH } \\
62080\end{array}$ & 6 & & 12 & NT & & II \\
\hline Cervidae & & & & & & & & \\
\hline Mazama americana (Erxleben) & Venado colorado, Puca luicho & $\begin{array}{l}\text { AMNH } \\
75957\end{array}$ & 6 & & & & & III \\
\hline $\begin{array}{l}\text { Mazama nemorivaga (F. Cuvier) } \\
\text { Delphinidae }\end{array}$ & Venado gris, Uchpaluicho & 10 & & & & & & \\
\hline $\begin{array}{l}\text { Sotalia fluviatilis (Gervais y Deville) } \\
\text { Iniidae }\end{array}$ & Bufeo gris, Bufeo negro & 4 & & & & & & I \\
\hline Inia geoffrensis (Blainville) & Bufeo colorado & $\begin{array}{l}\text { FMNH } \\
55517\end{array}$ & & & & & & II \\
\hline Total Órdenes & 11 & 10 & 7 & 5 & 5 & 9 & 7 & 8 \\
\hline Total Familias & 35 & 34 & 14 & 11 & 7 & 13 & 12 & 19 \\
\hline Total Especies & 192 & 189 & 16 & 39 & 10 & 20 & 17 & 44 \\
\hline
\end{tabular}

${ }^{1}$ Departamento de Mastozoología, Museo de Historia Natural, Universidad Nacional Mayor de San Marcos, Apartado 14-0434, Lima-14, Perú. Correo electrónico: heidi.qn@gmail.com / vpachecot@unmsm.edu.pe / salas.edith@gmail.com

2 Instituto de Ciencias Biológicas “Antonio Raimondi”, Facultad de Ciencias Biológicas, Universidad Nacional Mayor de San Marcos, Lima, Perú. 